\title{
Observational properties of a kink unstable coronal loop
}

\author{
M. Haynes and T. D. Arber
}

\author{
Centre for Fusion, Space and Astrophysics, Physics Department, Warwick University, Coventry, CV4 7AL, UK \\ e-mail: martin .haynes@warwick .ac .uk
}

Received 12 December 2006 / Accepted 12 February 2007

\begin{abstract}
Aims. Previous work on the dynamics of the kink instability has concentrated on the evolution of the magnetic field and associated current sheets. Here we aim to determine the observational consequences of the kink instability in short coronal loops, particularly what images TRACE would record of such an instability. This paper concentrates on the internal $m=1$ mode where the kink structure of the instability may not be apparent from the global field shape. This is most relevant to the observation of active region brightenings and coronal bright points.

Methods. An existing fluid code was modified to include the TRACE temperature response function in order to calculate temporally and spatially averaged, line of sight images in the 171, 195 and $284 \AA$ A band passes for straight, kink unstable flux tubes.

Results. Two new fluid effects of the kink instability are discovered: the circular enhancement of the density at the foot points and the appearance of a low density band running across the flux tube. The second of these effects is shown to be imagable by TRACE and hence would be a good candidate observational signature for an internal $m=1$ kink unstable loop.
\end{abstract}

Key words. Sun: corona - Sun: magnetic fields - Sun: flares - Sun: UV radiation

\section{Introduction}

One of the main uses of computer simulations of the solar corona should be to link theory with observation. Including diagnostics which allow the generation of observable quantities and images enables the output from theoretical models to be more easily compared to the output from telescopes. Over the past decade there have been numerous numerical studies of kink unstable loops in the solar atmosphere. Initially these concentrated on the straight flux tube approximation for computational simplicity and comparison with analytic theory. More recently simulations have begun to explore the effects of curvature on loops. For unstable eruptive loops, as associated with CMEs, the magnetic fields are possibly traced out in observations by cold, absorbing material. A direct comparison of field line topology between simulations and observations is therefore possible. One question which has however received little attention is what the unstable confined loops, with lengths of the order of tens of Mm, would look like in satellite images. For the short, hot loops considered in this paper the observations are of emission in EUV or soft $\mathrm{X}$-ray and to enable comparison with observations simulations must produce synthetic EUV images.

Previous theoretical work on straight flux tubes has looked in depth at various aspects of the kink instability in a solar context including the formation of current sheets (Baty \& Heyvaerts 1996; Gerrard et al. 2001; Gerrard \& Hood 2003), the magnetic topology (Baty 2000) and how the twist affects the kink evolution (Lionello et al. 1998). Also how the amount of twist in the instability compares with the theoretical critical twist for the Gold-Hoyle equilibrium of $2.49 \pi$ (Hood \& Priest 1981), where the twist is defined as $\Phi=L_{z} B_{\theta}(r) / r B_{z}(r)$ (Einaudi \& van Hoven 1983; Mikić et al. 1990). All of these works looked at the nonlinear growth phase and stopped when the initial helical current sheet had dissipated. For curved flux tube models previous work has addressed how the current sheets formed (e.g. Török et al. 2004), how the loops responded to twist being injected through foot points (Van Hoven et al. 1995; Amari et al. 1996; Amari \& Luciani 1999; Klimchuk et al. 2000; Török \& Kliem 2003; Tokman \& Bellan 2002; Gerrard et al. 2004; Aulanier et al. 2005) and how kink unstable loops erupt through overlaying arcades (e.g. Török \& Kliem 2005; Fan 2005). Some of these works also looked at the final magnetic field structure and compared these structures to loop images through field line plots (e.g. Tokman \& Bellan 2002; Török \& Kliem 2005; Fan 2005). For large magnetic features this approach has been successful in showing the similarity between observed field structures and those simulated. In 3D loop simulations it has been shown that the loop can expand upwards in the atmosphere depending on the nature of the overlying field (Török \& Kliem 2005; Birn et al. 2006). In this paper we concentrate on examples of short loops undergoing the kink instability in which the mode remains confined in space, the $m=1$ internal kink mode. This process has been shown (Arber et al. 1999) to release sufficient energy to cause a transient brightening of confined loops. Such loops are hot and observed in emission in EUV or soft X-ray. Since such loops are only likely to remain confined when the overlying field is strong we restrict our attention to short coronal loops. The loop model used in this paper is a loop which carries no net current, as might arise due to externally driven twisting motion. This has been found, through numerical simulation (see Arber et al. 1999), to keep the instability confined as compared to the equilibrium used in CME eruption papers (e.g. Török \& Kliem 2005), which lead to a global, external kink instability.

Coronal loops of similar length to the one simulated in this work $(10 \mathrm{Mm})$ have been observed as active region brightenings or coronal bright points. Initially in X-ray (e.g. Shimizu et al. 1994) and then with EIT and TRACE (e.g. Brown et al. 2001; 
Seaton et al. 2001; Berghmans et al. 2001). Brown et al. (2001) speculated that the bright point they studied might have gone kink unstable but the only diagnostic available was the amount of twist injected into the loop. Seaton et al. (2001) interpret the event studied in their work as a loop heating event and Shimizu et al. (1994) interpret theirs as sub-resolution loops interacting. Berghmans et al. (2001) looked at the correlation between the simultaneous X-ray, EIT and TRACE images. They also looked at the statistical distribution without speculation as to the underlying mechanism.

The Coronal loops seen by narrow band telescopes are highly temperature dependent and just comparing field line plots to TRACE images, see Handy et al. (1999), may be an oversimplification. It makes the assumption both that the loop has the correct temperature for that band pass and that there is sufficient mass in the loop to provide the emission. Getting the temperature correct is therefore critical to the successful generation of synthetic TRACE images. Previous work on CME eruption with long loops have used zero $\beta$ codes, (e.g. Török \& Kliem 2005; Aulanier et al. 2005) which give no information about the temperature in the corona. For the short, hot loops considered here we need to simulated the emission and therefore must have an acurate measure of the plasma temperature and the zero $\beta$ model is inappropriate. In this work we therefore use a Lagrangian-remap code (Arber et al. 2001) which is written in non-conservative form, while still capturing shocks. Using nonconservative form allows better modeling of the temperature in low plasma $\beta$ compared to conservative form as the pressure, and hence temperature, is calculated directly and not derived from the difference of two large terms, see Arber et al. (2001) for examples and details.

Some previous work has tried to obtain a more accurate impression of how a telescope might see the simulation. These have included line of sight integration of the Ohmic heating term to give an idea of what would be seen in soft X-ray (Aulanier et al. 2005) and the inclusion of an EIT response curve to give the emission from a 3D active region subject to different heating profiles (Mok et al. 2005). The kink instability is inherently 3D and hence the calculation of ionisation levels is too computationally expensive. In this paper we have therefore concentrated solely on line of sight and exposure averaging of the data combined with the TRACE response curves.

As a first step at simulating observational EUV emission from flaring loops only the straight tube kink instability is considered. The observable image is generated by modifying the output of the fluid simulation to include the emission as seen by TRACE. This allows the construction of synthetic TRACE images of the simulation giving a better understanding of what might actually be observable. This work continues that of Arber et al. (1999), hereafter Paper I, using the same initial conditions and resistive model. In Paper I it was shown that time and space averaging of the velocities, as might be interpreted from a spectroscopic Doppler shift, can change the observed quantities by significant amounts. The synthetic observables presented here therefore also include the time and space averaging to take into account the resolution and integration time of the TRACE telescope.

This paper is divided into the following sections: Sect. 2 gives an overview of the core fluid solver and how this is modified to include the emission, Sect. 3 describes the initial conditions, Sect. 4 describes various features of the simulation and Sect. 5 shows the associated TRACE image. The conclusions are then summarised in Sect. 6.

\section{Techniques}

\subsection{The fluid solver}

All the simulations were performed using Lare3D, see Arber et al. (2001). This code solves the resistive MHD equations in normalised Lagrangian form:

$$
\begin{aligned}
\frac{\mathrm{D} \rho}{\mathrm{D} t} & =-\rho \nabla \cdot \boldsymbol{v} \\
\frac{\mathrm{D} \boldsymbol{v}}{\mathrm{D} t} & =\frac{1}{\rho}(\nabla \times \boldsymbol{B}) \times \boldsymbol{B}-\frac{1}{\rho} \nabla P \\
\frac{\mathrm{D} \boldsymbol{B}}{\mathrm{D} t} & =(\boldsymbol{B} \cdot \nabla) \boldsymbol{v}-\boldsymbol{B}(\nabla \cdot \boldsymbol{v})-\nabla \times(\eta \nabla \times \boldsymbol{B}) \\
\frac{\mathrm{D} \epsilon}{\mathrm{D} t} & =-\frac{P}{\rho} \nabla \cdot \boldsymbol{v}+\frac{\eta}{\rho} j^{2} .
\end{aligned}
$$

Where $\rho, \epsilon, \boldsymbol{v}, \boldsymbol{B}, \eta$ are respectively the density, specific internal energy, velocity, magnetic field and resistivity. The pressure is given by $P=\rho \epsilon(\gamma-1)$ where $\gamma=5 / 3$ is the ratio of specific heats. The current density is given by $\boldsymbol{j}=\nabla \times \boldsymbol{B}$. These variables are normalised to $\rho_{0}, \epsilon_{0}, v_{A}=B_{0} / \sqrt{\mu_{0} \rho_{0}}, B_{0}$ and $\eta_{0}=\left(\mu_{0} L_{0} v_{A}\right)$. For all simulations the resistivity is of the form

$\eta=\left\{\begin{array}{l}0 \\ 1 \times 10^{-3},|j|<5\end{array}\right.$

These values are the same as used in Paper I. Whilst this value of resistivity is orders of magnitude larger than that expected in the solar corona it is the smallest that can be run in $3 \mathrm{D}$ and allow convergence testing. All of the results presented in the figures were generated on a $128^{3}$ grid which is uniform in $z$ but stretched in $x$ and $y$ to give more points in the region $|x|,|y|<1$. In the central region of interest this gives a resolution of $\Delta x=\Delta y=$ 0.031 and $\Delta z=0.078$. Convergence tests were run with $256^{3}$ resolution. One test used Eq. (5) while a second test modified Eq. (5) so that the anomalous resistivity was only triggered when $|j| \geqslant 10$ confirming that the peak current scales as $1 / \Delta x$. Both tests confirmed the results presented in this paper.

The normalisation used for this simulation are: lengths are normalised to $1 \mathrm{Mm}$, magnetic fields to $20 \mathrm{G}$ and densities to $1.67 \times 10^{-15} \mathrm{~g} \mathrm{~cm}^{-3}$. This gives a normalising Alfvén speed of $1.38 \times 10^{8} \mathrm{~cm} \mathrm{~s}^{-1}$ and therefore normalised time units are approximately $1 \mathrm{~s}$.

\subsection{Generating synthetic images}

In order to generate the synthetic images from TRACE the code needs to output the emission in the various bands. Here we are only using the 171, 195 and $284 \AA$ band passes. The TRACE response curves for these three bands were generated using the SolarSoft ${ }^{1}$ software. The response curves are normalised to their maximum value and are shown in Fig. 1. They were generated using the "feldman_raymond_pe1e15.resp" response file provided with the SolarSoft package. These response curves are included in the simulation code as a look up table providing a temperature scaling for $\rho^{2}$ which is then taken to be the emission. The value of the emission is calculated for every cell in the simulation after every time step. For this paper we have restricted the viewing position to be along either the $x$ or $y$ axis, viewing along the $z$ axis would be unphysical as that would be the equivalent of looking from one foot point to the other along the loop.

\footnotetext{
${ }^{1}$ http://www.lmsal.com/solarsoft/
} 


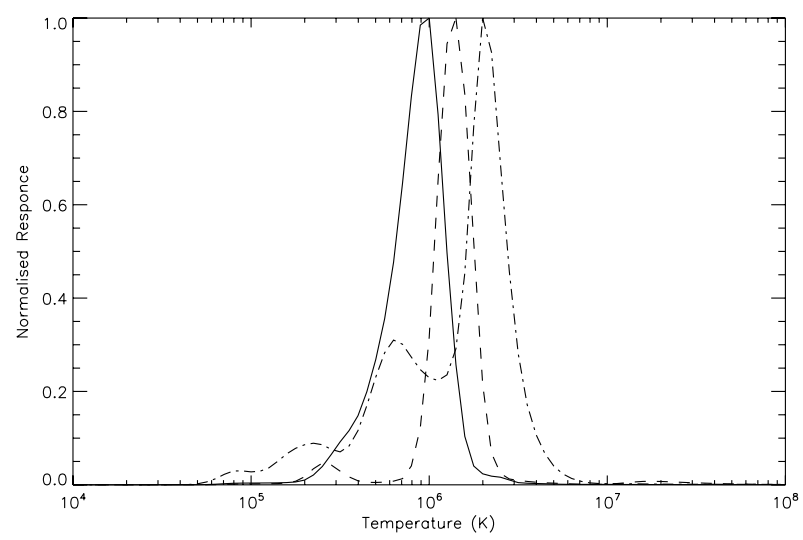

Fig. 1. The normalised temperature response curves used in the code to scale $\rho^{2}$ to give the emission seen by the different TRACE band passes. The solid line shows $171 \AA$, the dashed line shows $195 \AA$ and the dotdashed line shows $284 \AA$.

The line of sight integral is taken simply as the sum through the domain

$I=\int g(T) \rho^{2} \mathrm{~d} l=\sum_{i=0}^{N} g(T) \rho_{i}^{2} \Delta l_{i}$

where $I$ is the measured intensity, $g(T)$ is the temperature response function and $l$ is the distance along the line of sight. The code outputs $g(T) \rho^{2}$ for every cell and the TRACE image is therefore formed using the discrete sum from cell 0 to $N$ through the computational domain.

Due to the integration time of TRACE the code emission values are also integrated in time. The code outputs the emission data every 5 internal time units, this is the emission integrated over this period. This corresponds to approximately $5 \mathrm{~s}$ in our normalisation. These time integrated emission outputs can then be added together to give longer integration times. Throughout this paper two of these outputs are added together to give an integration time of approximately $10 \mathrm{~s}$. This value was chosen as it is the smallest integration time TRACE observes with and the phenomena being simulated are evolving on a similar time scale so a longer integration period would give very few images over the simulation evolution. The effect of the time integration can be seen in Fig. 2.

TRACE also integrates over space due to its pixel size. For the rest of the paper the pixel size is $0.36 \mathrm{Mm}$ at the Sun and the time averaged emission images are therefore also integrated over this spatial extent. Figure 2 shows the effect of this spatial integration. Both of these integrating processes remove small and quickly evolving features from the simulation data.

\section{The initial conditions}

The initial conditions are the same as those used in Paper I but with a density enhancement along the axis of the loop in order to make it visible in the TRACE emission diagnostics. The density enhancement is not in equilibrium but since this is low $\beta$ the departure from equilibrium will be slow compared to the velocities resulting from the magnetic instability. The initial temperature in the domain is uniform and set to 0.01 in normalised units, which corresponds to $1 \mathrm{MK}$.
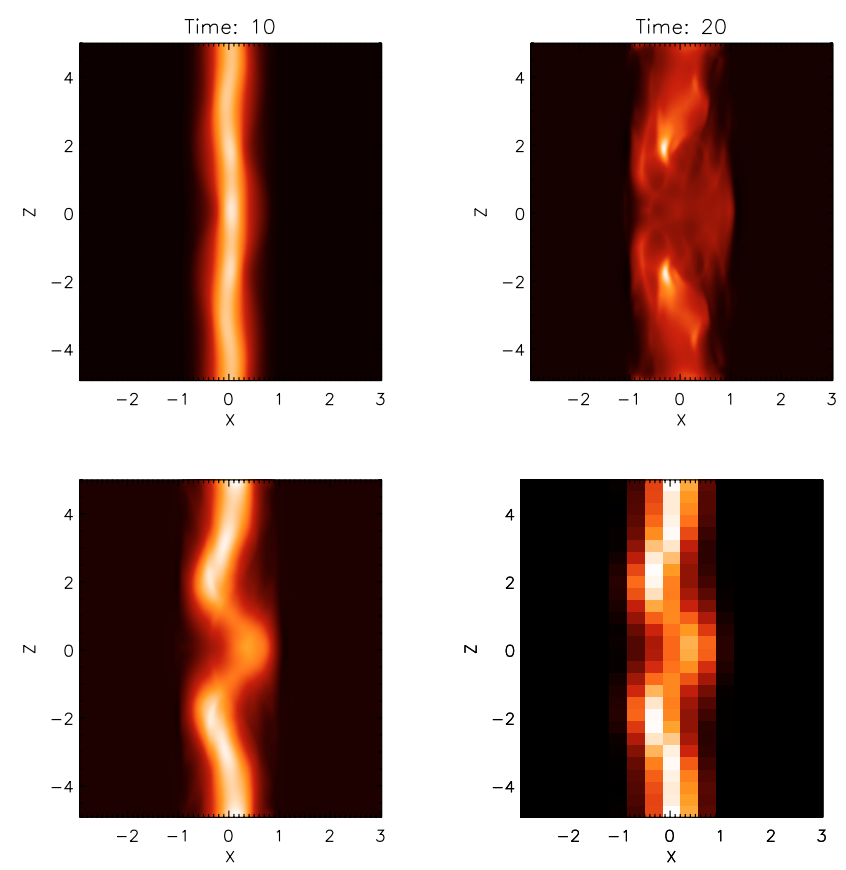

Fig. 2. Figure showing the effect of space and time averaging. The top two plots show line of sight (LoS) integrated $\rho^{2}$ through the domain along the $y$ axis at two different snapshots. Bottom left shows the effect of integrating the $\operatorname{LoS} \rho^{2}$ in time between these two snapshots. Bottom right shows the effect of spatially integrating the time averaged $\operatorname{LoS} \rho^{2}$.

The initial magnetic field is determined from a force-free cylinder that carries no net current and is defined in terms of the axial current density $j_{z}$ by

$j_{z}=j_{i}\left(1-\frac{r^{2}}{b^{2}}+a \frac{r^{3}}{b^{3}}\right)$.

The loop is confined within radius $r=1$ so that the free parameters $a$ and $b$ can be found from the condition that there is no current at $r=1$ and that it carries no net current, $\int_{0}^{r} r j_{z}(r) \mathrm{d} r=0$. The values used are therefore $a=5 / 6^{3 / 2}$ and $b=1 / 6^{1 / 2}$. The poloidal component of the magnetic field, $B_{\theta}$, is found from Ampere's law and $B_{z}$ from the force-free condition

$B_{z}^{2}=B_{i}^{2}-B_{\theta}^{2}-2 \int_{0}^{r}\left(\frac{B_{\theta}^{2}}{r^{\prime}}\right) \mathrm{d} r^{\prime}$.

The values of $j_{i}$ and $B_{i}$ are therefore free parameters and are chosen to be 1.0 and 4.3 respectively.

The density enhancement in the loop, $r \leq 1$, is given by

$\rho=\rho_{i}(1+\cos (\pi r))+\rho_{\mathrm{b}}$

where $\rho_{i}$ scales the size of the density enhancement and $\rho_{\mathrm{b}}$ is the background density. These values are set to $\rho_{i}=0.45$ and $\rho_{\mathrm{b}}=0.1 . \rho$ is set to $\rho_{\mathrm{b}}$ outside $r=1$. This enhancement leads to a peak plasma beta in the flux tube of 0.03 .

To assess the impact of the density enhancement on the system the simulation was also run with a uniform density of $\rho=0.1$. The evolution of the system followed the same stages as presented later, however the evolution was of course faster due to the increased Alfvén speed in the loop. The generated TRACE images were, as expected, less well defined due to small density perturbations outside the tube having similar intensity to those inside. 


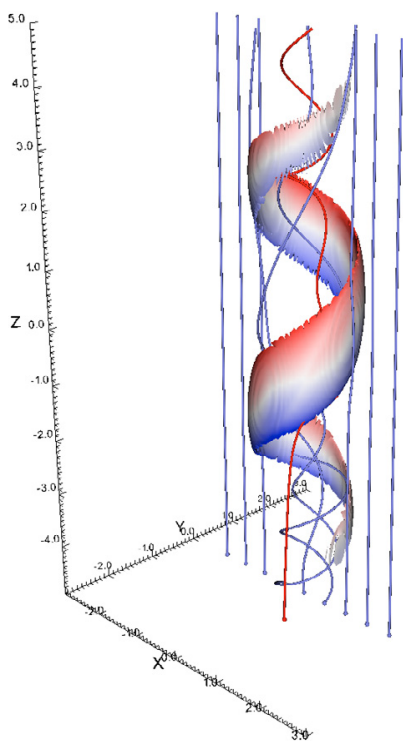

Fig. 3. Snapshot from the simulation at time 13. The isosurface is of $|j|=5$ and coloured by the vertical velocity component at the surface. Red shows upward directed flow and blue downward. The blue field lines are traced from the bottom boundary along the $x$ axis. The red field line shows a field line which starts outside the original tube and end inside.

Since the initial flux tube is in the centre of the simulation domain and the kink instability is well confined in radius the $x$ and $y$ boundaries do not influence the evolution. The domain boundaries in $x$ and $y$ are placed at \pm 3 and are set by projecting the 1D Riemann invariants along characteristics with the inflowing characteristics specified by the far-field solution, i.e. the equilibrium above defined in the limit of large radius. This gives an open boundary by updating the ghost cells using either the characteristic waves from the internal solution if they are outward propagating or the characteristic waves from the far-field if they are inward propagating. This reduces noise at the boundary and allows outward propagating waves to leave the domain. The $z$ boundary is placed at \pm 5 and is line tied giving a loop length, $L_{z}$, of 10 . Through the definition of $B_{i}$ and $j_{i}$ above this places the average twist in the loop above the critical value of $2.49 \pi$.

\section{Simulation dynamics}

Figure 3 shows a snapshot from the simulation showing the formation of a current sheet in a helix around the tube axis. The initial $m=1 \mathrm{kink}$ instability causes the loop to writhe with the largest displacement at the centre of the domain. The $m=1$ instability is helical and a current sheet forms at the radius where the helical twist of the linear instability $\left(L_{z} k\right)$ matches the twist $(\Phi)$ of the equilibrium magnetic field (the mode rational surface), where $k$ is the axial wavenumber of the instability. The formation of current sheets, and their location, is complex for line-tied equilibria (e.g. Gerrard \& Hood 2003; Baty \& Heyvaerts 1996) but has been verified for the equilibrium considered in this paper (Arber et al. 1999). The current sheet magnitude increases until it exceeds the trigger value for the anomalous resistivity, at which point reconnection occurs. The high twist tube field lines reconnect with the outer, low twist field lines. The new field lines formed by this reconnection process have one high twist end in the core of the tube and one almost straight end external to the tube.
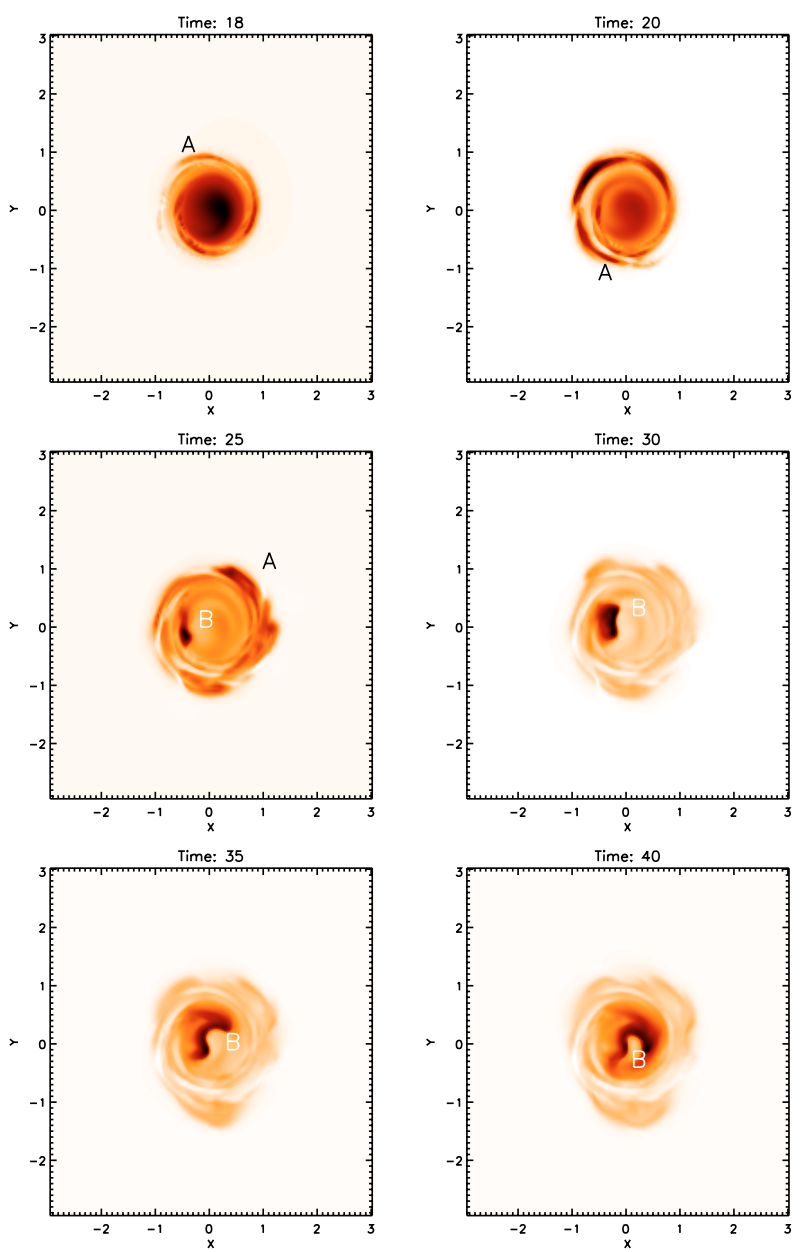

Fig. 4. Series of images showing the evolution of the density in a slice through the loop foot point at $z=-5$. "A" marks the leading edge of the outer anti-clockwise evolving density enhancement, "B" shows the inner clockwise enhancement.

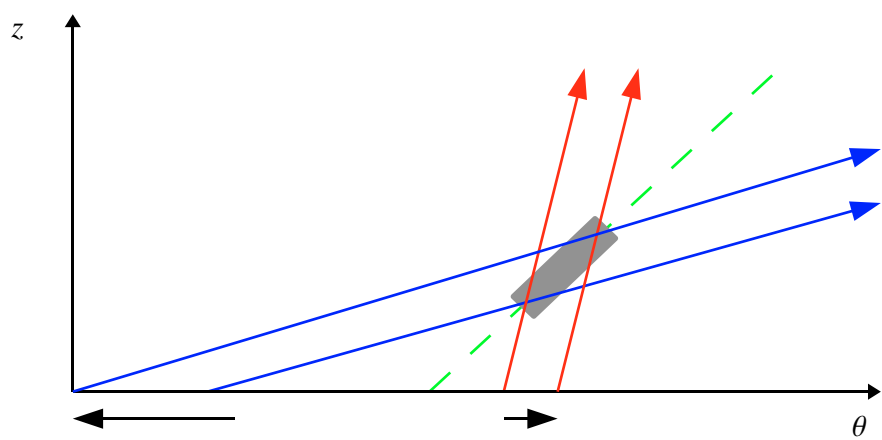

Fig. 5. Cartoon diagram showing how a single reconnection region can cause time delayed, oppositely evolving density enhancements. The tube has been unrolled at the rational surface to form a plane. The green dashed line shows the direction of a field line on the rational surface where the reconnection occurs. The red lines are the field lines initially external to the surface projected down to the plane and the blue lines are the twisted internal field lines projected up onto the plane. The grey area shows the reconnection region and the black arrows show the direction in which the density enhancements are observed to move at the foot points.

Plasma which has been heated both by the compression driving the reconnection and the Ohmic heating as it passes through the reconnection region now expands along the new field lines. 

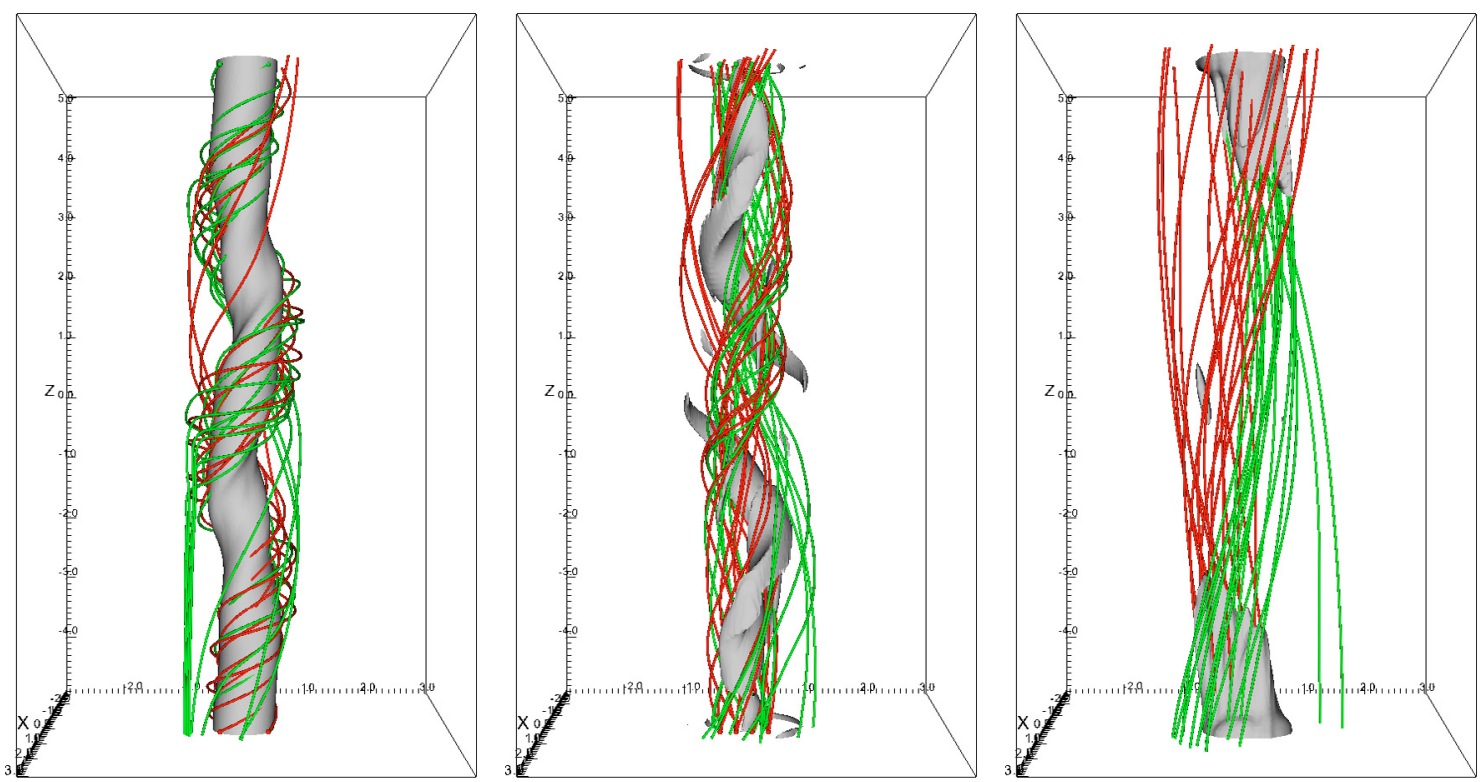

Fig. 6. Series of snapshots from the simulation at normalised time 13, 20 and 100 . The isosurface is of normalised density equal to 0.6 . The red field lines are traced from the bottom boundary within a radius of 0.5 . The green field lines are traced backwards from the top boundary within a radius of 0.5 .

The consequences of this for the foot points can be seen in Fig. 4. There is an initial brightening outside the rational surface moving in an anticlockwise direction for the lower footpoint. The foot point in Fig. 4 is at the bottom of the domain and as can be seen from the orientation of the current sheet shown in Fig. 3 the plasma flowing straight down from this sheet would, due to time of flight, cause a bright spot to start on the positive $y$ side of the tube and stretch round the loop anticlockwise. The bright spot is caused by the plasma being compressed against the photosphere, in this case the line tied boundary. The condition on the density for the line tying is zero gradient perpendicular to the boundary and the boundary velocities are set to zero. The density increase occurs over a range of $3-4 \%$ of the flux tube length so the exact form of the boundary conditions does not matter, so long as it impedes the plasma flow.

The central brightening then starts to form closer to the loop axis, but rather than also extending round the tube anticlockwise, it instead extends clockwise round the foot point. The mechanism for this can be explained using Fig. 5. The helical current sheet forms rapidly along most of the length of the rational surface, due to line tying of the ends it does not extend completely to the foot points. Reconnection therefore commences along the length of the current sheet at approximately the same time. As can be seen from Fig. 5 if the grey region were to reconnect at exactly the same time, with the reconnection outflow directed along the new field lines, the lengths of these new field lines will give the time for the outflow to reach the foot points. Taking the lower axis to be a foot point, the order would then be as follows. The left hand red field line is the shortest and would therefore brighten first followed by the right hand red field line. As the red lines are external to the rational surface this gives a brightening progressing in the same direction as the twist projected onto the foot point. The next field line to brighten is the right hand blue field line followed by the left hand blue field line. As can be seen this gives the brightening appearing in a direction opposing the direction of the twist. It also proceeds slower than the external brightening due to the larger difference in line length.

The reconnection leaves the field lines with one end containing high twist and one low twist. The high twist region is in the core of the tube and the low twist outside the original tube. Having one foot point connected to the external region and the opposite foot point connected to the region inside the original rational surface means that the untwisting of this field line involves a radially, outward motion of plasma. This effect is most pronounced at the loop apex. This can be seen in Fig. 6 which shows field lines which start on the bottom foot point inside the rational surface, the red lines, and those connected to the upper foot point inside the same initial rational surface, the green lines. The unwinding of the field at $z=0$, the loop apex, leads to an a reduction in density in a diagonal band across the loop. This continues until at $t=100$ the entire loop is at a lower density. The field slowly relaxes to the state shown in the final snapshot in Fig. 6. This state is the same topologically as the final state given in Baty (2000), see Fig. 6 therein for a comparison.

\section{Observation results}

Attention is now turned to how TRACE would see this dynamic evolution. Figure 7 shows how TRACE would view the loop going unstable. It can be seen that the loop heats predominantly in the centre as it fades from the $171 \AA$ images producing bright spots in the higher band passes due to the compression caused by the loop kinking and the subsequent Ohmic heating. The formation of the diagonal gap due to the field lines untwisting after the reconnection can be clearly seen in all three band passes as a dark line running through the tube. This is because this region is of a lower density than the rest of the tube, most of the original tube density is still confined within the high twist regions. This diagonal feature is good candidate for a kink instability signature.

Figure 8 shows the long term evolution of the loop in the three different band passes. The loop fades from $171 \AA$ and then from both $195 \AA$ and $284 \AA$ as it heats up. The heating of the central plasma and subsequent compression of the foot points causes foot point brightening without there being any foot point heating source. 

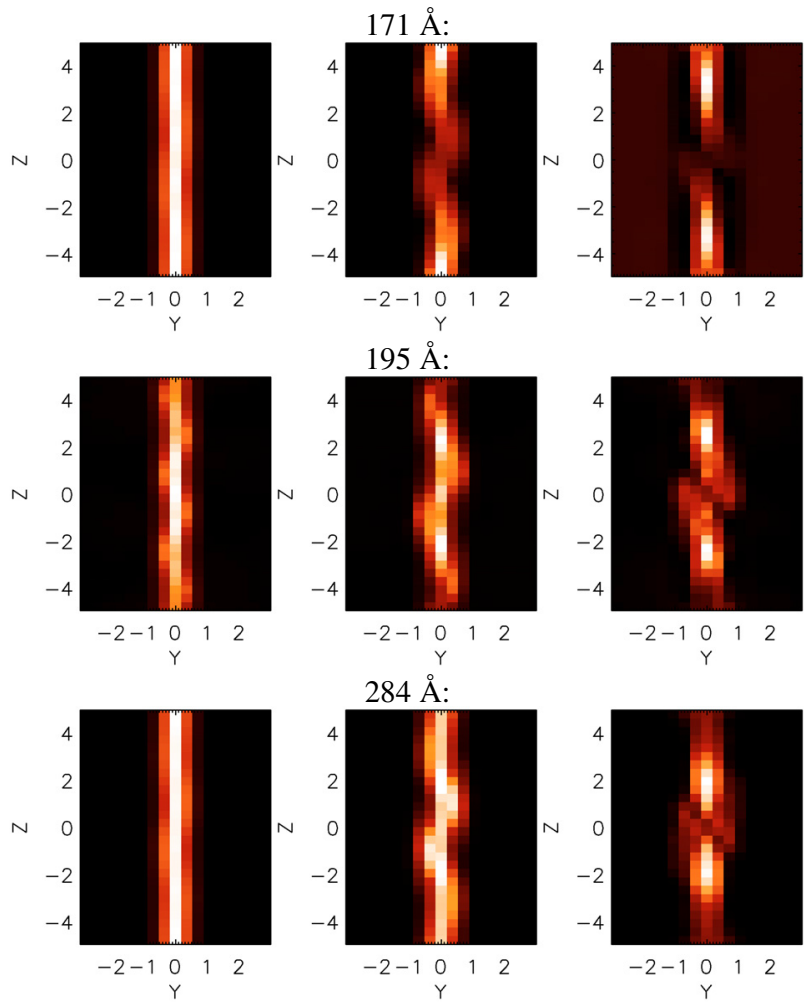

Fig. 7. Series of images of the loop during the non-linear phase. From left to right the columns shows times 10,20 , and 30 . The images are from the three band passes integrated in space and over the previous 10 normalised time units. Brighter colours corresponding to regions of greater intensity.
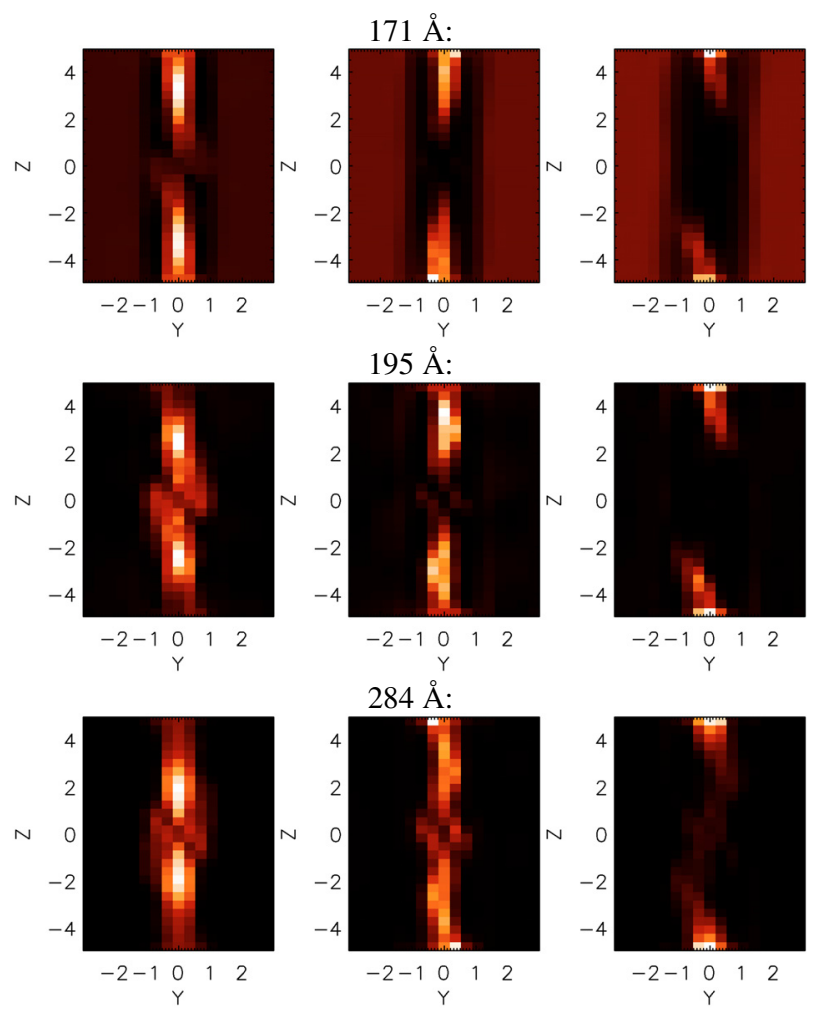

Fig. 8. Series of images in the three different bands at times, from left to right, 30, 40 and 50. All are spatially integrated and time integrated over the previous 10 internal time units.
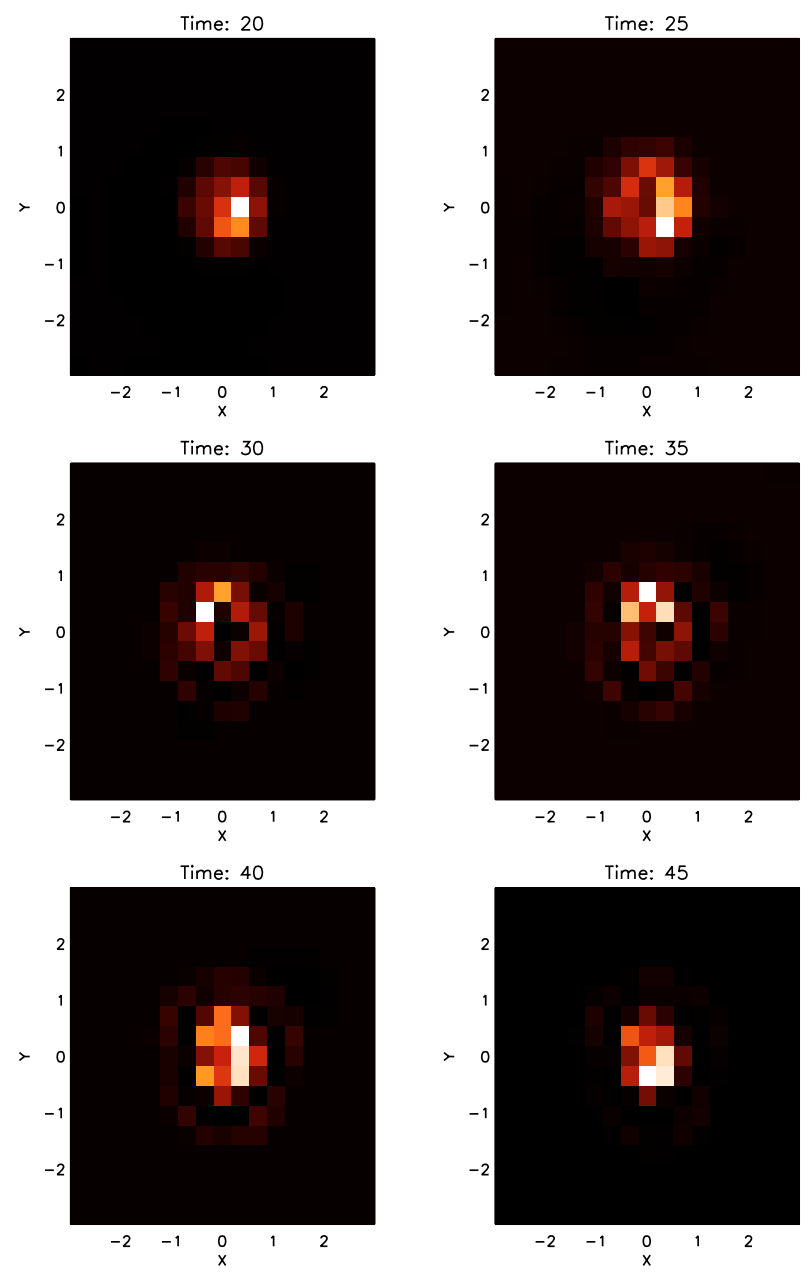

Fig. 9. The same sequence of images as seen in Fig. 4 shown in the $195 \AA$ A band pass. These all include time and space integrals, in the plane of the image, but are not line of sight integrated.

In Fig. 4 another property of the helical current sheet associated with the kink instability was presented which is the evolution of a density increase around the loop axis at the foot point. Figure 9 gives an approximation of what might be observed. One of the shortcomings of using the straight loop model is the inability to do the line of sight integration looking down onto the loop foot points. Figure 9 is therefore a time and space integrated slice through the foot points rather than a line of sight observation. However, as can be seen the spatial resolution available to TRACE is not sufficient to resolve all of the features of this foot point brightening.

\section{Conclusion}

This work address the question of what observational signatures would be present if one were to look for evidence of short, high aspect ratio loops going kink unstable. In doing so a more complete picture of the actual fluid consequences of the kink instability were investigated leading to the following:

- The reconnection causes flows directed towards the foot points which, due to time of flight effects, cause a density increase to appear outside the rational surface which evolves by extending around the loop in the direction of the twist. A second density enhancement then forms on the inside of the rational surface and evolves in the opposite direction to the 
twist. This would be a good candidate for a signature of the kink instability however it is probably just below the spatial resolution currently available. As shown in Fig. 9 some motion of the location of peak emission may be detected but not all of the structure described above.

- The reconnecting of the internal field lines to the external field lines generates field lines which are straight from one foot point to the reconnection site and then twisted from that site to the other foot point. The motion associated with the untwisting of these field lines leads to a density depletion diagonally across the loop axis.

- The splitting in half of the tube causes a diagonal density depletion which when viewed from the $x$ direction, corresponding to an observation of a loop on the solar disk, is a good candidate for the kink instability signature. This density depletion should also be visible in TRACE images as it shows up quite clearly in Fig. 7.

An additional observational characteristic of an internal $m=1$ instability driving reconnection in a compact loop was also reported in Paper I which should be combined with the above in any complete assessment of the kink instability. This was that there will be exposure and line of site averaged Doppler measurable flow away from the loop apex of around 40-100 $\mathrm{km} \mathrm{s}^{-1}$.

The simulations presented here are limited in that the flux tube is initially straight and thermal conduction, along with any subsequent chromospheric evaporation, has been ignored. The magnitudes of the time averaged velocities and appearance of a dark band across the loop apex ought to be robust. This is because these features occur early in the simulation and are fast thereby avoiding any influence from conduction or evaporation. The extent to which the foot point brightening would be observed as described here once chromospheric evaporation is included remains an open question and will be the subject of future study.
Acknowledgements. This work was sponsored in part by the UK Particale Physics and Astronomy Research Council. The computational work was support by resources made available through the UK MHD Consortium and Warwick University's Centre for Scientific Computing.

\section{References}

Amari, T., \& Luciani, J. F. 1999, ApJ, 515, L81

Amari, T., Luciani, J. F., Aly, J. J., \& Tagger, M. 1996, ApJ, 466, L39

Arber, T. D., Longbottom, A. W., \& Van der Linden, R. A. M. 1999, ApJ, 517, 990

Arber, T. D., Longbottom, A. W., Gerrard, C. L., \& Milne, A. M. 2001, J. Comput. Phys., 171, 151

Aulanier, G., Démoulin, P., \& Grappin, R. 2005, A\&A, 430, 1067

Baty, H. 2000, A\&A, 360, 345

Baty, H., \& Heyvaerts, J. 1996, A\&A, 308, 935

Berghmans, D., McKenzie, D., \& Clette, F. 2001, A\&A, 369, 291

Birn, J., Forbes, T. G., \& Hesse, M. 2006, ApJ, 645, 732

Brown, D. S., Parnell, C. E., Deluca, E. E., Golub, L., \& McMullen, R. A. 2001, Sol. Phys., 201, 305

Einaudi, G., \& van Hoven, G. 1983, Sol. Phys., 88, 163

Fan, Y. 2005, ApJ, 630, 543

Gerrard, C. L., \& Hood, A. W. 2003, Sol. Phys., 214, 151

Gerrard, C. L., Arber, T. D., Hood, A. W., \& Van der Linden, R. A. M. 2001, A\&A, 373, 1089

Gerrard, C. L., Hood, A. W., \& Brown, D. S. 2004, Sol. Phys., 222, 79

Handy, B. N., Acton, L. W., Kankelborg, C. C., et al. 1999, Sol. Phys., 187, 229

Hood, A. W., \& Priest, E. R. 1981, Geophys. Astrophys. Fluid Dyn., 17, 297

Klimchuk, J. A., Antiochos, S. K., \& Norton, D. 2000, ApJ, 542, 504

Lionello, R., Velli, M., Einaudi, G., \& Mikić, Z. 1998, ApJ, 494, 840

Mikić, Z., Schnack, D. D., \& Van Hoven, G. 1990, ApJ, 361, 690

Mok, Y., Mikić, Z., Lionello, R., \& Linker, J. A. 2005, ApJ, 621, 1098

Seaton, D. B., Winebarger, A. R., DeLuca, E. E., et al. 2001, ApJ, 563, L173

Shimizu, T., Tsuneta, S., Acton, L. W., et al. 1994, ApJ, 422, 906

Tokman, M., \& Bellan, P. M. 2002, ApJ, 567, 1202

Török, T., \& Kliem, B. 2003, A\&A, 406, 1043

Török, T., \& Kliem, B. 2005, ApJ, 630, L97

Török, T., Kliem, B., \& Titov, V. S. 2004, A\&A, 413, L27

Van Hoven, G., Mok, Y., \& Mikić, Z. 1995, ApJ, 440, L105 